\title{
EDITORIAL
}

\section{ON THE BIRTH OF THIS JOURNAL}

It is an exciting time for "micro" technology. Authors working in microlithography, microfabrication, and microsystems finally have a home journal. Their work will be collected, peer reviewed, published, and archived. Most important, when literature in this field is needed, there is a clear place to locate it.

Launching a new journal is not easy. Starting in 2000, SPIE took action to turn its long-time vision into reality. After approval of the proposed plan near the end of the year, a search committee was formed for the journal editor. Subsequently I was contacted. I accepted the challenge in April 200I and the editorial board was established in June. A Call for Papers was sent at that time, nine months before the time you are reading this editorial. All papers had to be sent to the typesetter by December 200I. That left us six months to receive the manuscripts, assign them to editors who in turn sent them to two or more reviewers, and collect the reviews. The manuscripts that survived rejection were revised and went for further review before final acceptance. Many manuscripts could not make the inaugural issue because of the tight schedule. We will keep striving to shorten the review cycle while upholding quality.

Now that the journal is a reality, it owes its birth to many people. First there are the SPIE leadership who had the vision to fulfill the needs of the micro technical community for an archival journal. Then there are the SPIE editorial staff who shared the experience they have accumulated from publishing other SPIE sponsored journals and provided invaluable support. The journal owes its soul to the authors, reviewers, editors and, most important of all, to you, the reader. Without you, who will enjoy the fruit of the hard work? Who will submit their manuscripts? Who will play the role of unsung heroes to review?

The official name of this newborn is the Journal of Microlithography, Microfabrication, and Microsystems. The abbreviated name is $\mathrm{JM}^{3}$. You may often find it written as JM3 because of situations where superscript fonts are not available. All three forms convey the unique synergy among microlithography, microfabrication, and microsystems.

Quality is what we look for in $\mathrm{JM}^{3}$. There is an abundance of written information in the $M^{3}$ fields. The information can be found in many conference proceedings as well as trade journals. What is missing is a selection mechanism to single out the ones that are innovative, useful, rigorous, and worthy of archiving, and a gathering mechanism to attract them to just one place. We, with the help of the reviewers, will work hard to achieve this.

We are aware that most $M^{3}$ people are extremely busy. We hope to publish their good work without burdening them further. This leads to considering proceedings articles for submission to the journal. However, there are several problems with this: (I) lowering of publication standards, (2) double counting of publications, and (3) copyright violations. Let me address these problems one by one.

Lowering of Publication Standards: Generally, proceedings articles are prepared as a snapshot to report one's work. This may lead to incompleteness, insufficient proof or theoretical basis, lack of thoroughness in citing references, and unpolished writing. However, it is not necessarily so. Many authors take pride in their proceedings articles and prepare them up to the standard of journal articles. We do not want to force these writers to artificially modify their work just for the sake of modification. Therefore, a manuscript will be judged by its worthiness for journal publication instead of its similarity to the proceedings counterpart. However, it is our experience that most authors find room to expand and polish their proceedings articles. We recommend that the authors review their proceedings work themselves objectively and make improvements if needed, before sending it to us.

Double Counting of Publications: Authors are requested to identify the proceedings article counterparts in their journal article and to cease citing their proceedings work as soon as the journal article is accepted for publication. Readers of the journal article are urged to follow the same practice.

Copyright Violation: Authors are required to secure the copyright, if their journal article is based on publication in a conference proceedings. It is best to improve the article substantially so that there is no copyright issue.

In short, $\mathrm{JM}^{3}$ will not turn away a manuscript that is identical to a proceedings article but will review the manuscript vigorously just as any nonproceedings manuscript. The authors are always required to avoid double counting and copyright violation. There are cases where a proceedings manuscript has the basic ingredients for archival journal publication. In such cases, I suggest that proceedings authors prepare their manuscripts according to journal standards and submit a journal article to $\mathrm{JM}^{3}$ and a proceedings article to the symposium simultaneously. This way, the proceedings publishers get their material on time and in better quality. $\mathrm{JM}^{3}$ benefits from printing good work much sooner. The authors save one approval process from their institution.

SPIE symposia that potentially contain appropriate papers for $\mathrm{JM}^{3}$ include:

Microlithography 
Photomask Technology

Photomask Japan

Micromachining, Micromanufacturing, and Microelectronic Manufacturing

Smart Structures and Materials

Microelectronics and MEMS Technologies

Optoelectronics

In this inaugural issue, there are papers on mask design, imaging, birefringence, resolution enhancement, anti-reflection coatings, thin film resonators, and micromirrors. There seem to be more papers in microlithography than microfabrication and microsystems. Actually, this is the result of faster review cycles facilitated by more editors and reviewers in the microlithography field. In the next issue, the balance may be tilted to the other fields. I would like to take this opportunity to encourage more people to volunteer as reviewers and editors in these two fields. Microlithography also needs volunteers to serve as reviewers.

$\mathrm{JM}^{3}$ will initially be published quarterly with the goal to progress to bimonthly then monthly as paper submissions build up. We are planning to publish special sections on "Lithography for the 100 and $70 \mathrm{~nm}$ nodes," "Sacrificial surface micromachining," "Mask technologies," and "Micro-optics for photonic networks" in addition to regular papers in upcoming issues. You are welcome to submit papers to these special sections as well as regular contributed papers. Manuscripts for the special section on "Lithography for the 100 and $70 \mathrm{~nm}$ nodes" should be submitted by I April to facilitate a planned October publication. See the call for papers in this issue.

Happy reading!

\section{Burn J. Lin \\ Editor-in-Chief}

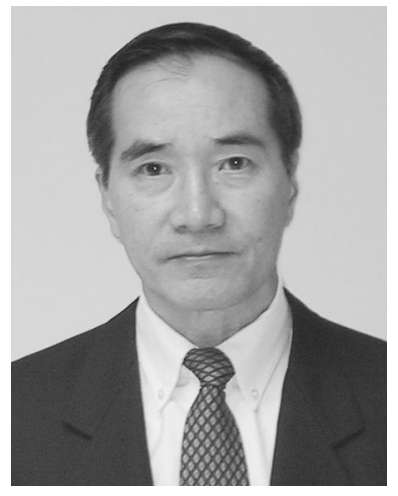

